\title{
Archaeal populations preferentially associated with recalcitrant dissolved organic matter in estuarine sediment
}

\author{
Wenxiu WANG ${ }^{1}$, Jianchang $\mathrm{TAO}^{2}$, Chen $\mathrm{HE}^{3}$, Quan $\mathrm{SHI}^{3}$, Penghui $\mathrm{LI}^{2}$, Hongmei $\mathrm{CHEN}^{4}$, Chuanlun
}

\section{$\mathrm{ZHANG}^{2 *}$}

${ }^{1}$ State Key Laboratory of Marine Geology, Tongji University, Shanghai, China (sophiawwx@163.com)

${ }^{2}$ Shenzhen Key Laboratory of Marine Archaea Geo-Omics, Department of Ocean Science \& Engineering,

Southern University of Science and Technology, Shenzhen, China (taojianchang@163.com,

lipenghui1987@126.com, ${ }^{*}$ correspondence: zhangcl@sustech.edu.cn)

${ }^{3}$ State Key Laboratory of Heavy Oil Processing, China University of Petroleum, Beijing, China

$$
\text { (hechen@cup.edu.cn, sq@cup.edu.cn) }
$$

${ }^{4}$ Department of Chemistry and Biochemistry, Old Dominion University, Norfolk, Virginia, USA

$$
\text { (hchen015@odu.edu) }
$$

Dissolved organic matter (DOM) in sediment pore water provides carbon substrates and energy sources for microbial populations driving benthic biogeochemical processes. Microbe-organic molecule interactions shape DOM and microbial community compositions, which are dynamic but elusive. In this study we characterized DOM composition in a 300-cm sediment core from the Pearl River estuary (China), in an effort to decipher its associations with relevant microbial communities. DOM composition showed a substantial decrease from surface to the bottom in the magnitude-weighted average $\mathrm{H} / \mathrm{C}$ ratio (from 1.48 to 1.17 ) and an increase in the magnitude-weighted average molecular weight (from $354 \mathrm{Da}$ to $418 \mathrm{Da}$ ). Molecules commonly representing recalcitrant organic matter were observed to accumulate in the deeper layers, which were characterized by lignins/carboxylic rich alicyclic molecules (CRAM)-like compounds, tannin and aromatics. DOM molecules correlated with most archaeal OTUs $(\mathrm{R}>0.65, \mathrm{P}$ $<0.05$ ) are of high $\mathrm{C}$ numbers, low $\mathrm{H} / \mathrm{C}$ ratios and high double bond equivalents compared to those correlated to bacterial OTUs, indicating that bacteria are associated with more relatively labile compounds while archaea may be closely related to refractory DOM. Our study contributes to the understanding of the sediment carbon dynamics and the differential roles of bacteria and archaea in organic matter degradation processes. 